Original Research Paper

\title{
A Theory that Wants to Obtain Particles Opposite to Protons, Having a Negative Charge
}

\author{
Relly Victoria Virgil Petrescu and Florian Ion Tiberiu Petrescu \\ ARoTMM-IFToMM, Bucharest Polytechnic University, Bucharest, (CE), Romania
}

\author{
Article history \\ Received: 23-08-2020 \\ Revised: 08-09-2020 \\ Accepted: 10-09-2020 \\ Corresponding Author: \\ Relly Victoria Virgil Petrescu \\ ARoTMM-IFToMM, \\ Bucharest Polytechnic \\ University, Bucharest, (CE), \\ Romania \\ Email: rvvpetrescu@gmail.com
}

\begin{abstract}
The present paper focuses on the possibility of obtaining antimatter, in the main purpose of then extracting massive energy by controlled fusion of matter and newly obtained antimatter. Since the most well-known charged nucleon (of the matter) is the proton, we imagined another possibility to obtain a particle opposite to it, having the same main characteristics but the opposite charge, i.e., negative and all this starting from a neutron and an accelerated electron. The particle thus obtained, although it has the opposite characteristics of a proton, does not resemble the antiproton imagined by physicists, which has all the quarks opposite to those of a proton, but will contain three quarks down. Such a particle will still be opposed to a proton and therefore likely to be able to annihilate with it when they meet so that it can be used to obtain massive energy by annihilation between protons and these opposite particles, which we will name negative protons.
\end{abstract}

Keywords: Proton, Neutron, Electron, Antiparticle, Nuclear Energy, A Down Quark, An up Quark, Hydrogen Proton, Kinetic Energy

\section{Introduction}

Antimatter is the term used to define the opposite of matter made up of protons, neutrons and electrons. In the same way that the term day defines both the $12 \mathrm{~h}$ period of light and the $24 \mathrm{~h}$ period that includes night, the term matter is used to define both the opposite of antimatter and all matter and antimatter in the universe. Antimatter consists of antiparticles. If the atoms that makeup objects used by humans are made up of protons, electrons and neutrons, the so-called anti-atoms will be made up of antiprotons, antielectrons (positrons) and antineutrons. The antiparticle differs from the particle in that it has a charge opposite to the particle, but has a mass equal to it. If a particle collides with its antiparticle, the two annihilate, emitting gamma rays, high-energy photons.

When matter and antimatter meet, they react violently. Matter and antimatter disappear (annihilate), leaving behind a form of energy, usually later stabilized as a highenergy photon (gamma rays). An Earth scrutiny of cosmic radiation could help detect such rays and thus identify a boundary zone between a realm of matter and antimatter. As this type of radiation has not yet been discovered at high intensities, areas of the Universe made up mostly of antimatter have not yet been discovered.

According to scientists, two equal amounts of matter and antimatter were created during the formation of the universe. So the two quantities should have annihilated each other. Due to an as yet unknown fact, this has not happened and the amount of antimatter in the universe is currently very low.

One second after the Big Bang, when the temperature was in the tens of billions of Kelvin, the universe contained mostly photons, electrons and neutrinos, as well as their antiparticles, but also protons and neutrons, in smaller quantities. Matter and antimatter coexisted without annihilating shortly after the Big Bang.

In the early universe, there was a balance between pairs of electrons and positrons that collided to create photons and the reverse process. They were annihilated continuously, generating light from which matter and antimatter were formed again. These phenomena - the creation of matter and antimatter starting from light and the annihilation of light generators - are observable in nuclear physics laboratories.

In that first second after the Big Bang, the amounts of matter and antimatter were about equal, with a very small difference. This difference was in favor of ordinary matter. Due to the cooling that occurred as a result of the expansion of the universe, matter and antimatter were annihilated without being reconstituted.

As the temperature of the universe dropped, the equilibrium shifted in favor of photon production. Eventually, most electrons and positrons in the universe 
were annihilated, leaving only relatively few electrons present today. Everything disappears, except for a small amount of matter. This rest results from the slightest numerical superiority of matter. It is the whole matter we know and the visible universe of today - galaxies, swarms, super-swarms, mega-swarms of galaxies.

As a result of thermonuclear reactions and the very high gravitational force exerted by stars, the process of concentrating matter develops. Consecutively, there is the appearance of pulsars, white dwarfs, neutron stars, there is, ultimately, the appearance and evolution of black holes, formations that concentrate stellar matter in the area of influence. In some areas of the universe where black holes appear, astral lines of force are created in the form of funnels that represent the directions of the absorption of cosmic matter.

According to some authors, this is where a maximum concentration of matter occurs which results in the appearance of neutron stars or black holes (Choi, 2008).

A possible explanation for the balance of matterantimatter could be based on the combined balance of positive-mass and negative-mass matter (which is thought to exist in the dark, massive matter in the universe), so there may be a balanced combination of matter. matter and antimatter, matter with positive mass and matter with negative mass, whose existence is today more and more suspected, being concentrated in the dark matter of the universe (Petrescu, 2020a).

Astrophysicists confirm that there is no significant amount of antimatter in the solar system, among the stars of the Galaxy, nor in neighboring galaxies. As for the possible existence of anti-galaxies at very distant distances, nothing can be said.

Antimatter can be produced on Earth in particle accelerators, either by the collision of subatomic particle beams with fixed targets, or with other particle beams, or by the collision of matter and antimatter (protons with antiprotons or electrons with positrons); by radioactive decomposition of atomic nuclei. Such a nucleus is used for the medical imaging technique called PET scanning or positron emission tomography; when high-energy cosmic particles (similar to those from the Sun called the solar wind) hit nuclei in the Earth's atmosphere. They annihilate very quickly with particles of matter around them, resulting in new particles or light (Hinshaw, 2010; Fritz Zwicky's Biography at the Zwicky Foundation, 2010; The British Universal Encyclopedia, 2010).

In August 2000, CERN laboratories in Geneva, Switzerland completed the construction of an "antimatter plant". Its purpose is to create antihydrogen atoms. The problem is that these atoms, once synthesized, can annihilate by contact with matter. This problem could be solved with the help of magnetic vacuum "traps", the socalled Penning traps, to prevent such collisions.

Small amounts of antimatter have been detected in an area of the Universe dominated by matter. Antimatter easily encounters the surrounding matter, annihilating it, resulting in gamma rays. This light has been detected since 1978 as coming from the center of our galaxy. Research has continued and now physicists are proposing a mechanism to explain the appearance of this antimatter. This antimatter exists for a very short time, quickly hitting matter and annihilating itself. Observing this light allowed astronomers to detect the presence of this antimatter (Choi, 2008).

Rockets, as they have evolved so far, can carry people per month and there is a possibility that they will reach other nearby celestial bodies in the future. But for the journey between two solar systems, chemical propulsion is not enough. To reach the nearest star using chemical propulsion, it would take 5 years of continuous travel at a speed comparable to the speed of light and therefore a very large amount of fuel.

It has recently been discovered that the energy produced by the annihilation of a small amount of matter with antimatter is much higher than that produced by the chemical process of combustion. A tiny amount of annihilated antimatter can provide a lot of energy, according to Albert Einstein's famous equation, $\mathrm{E}=\mathrm{mc} 2$, which also increases its financial value (Trimble, 1987).

In astronomy and cosmology, dark matter is currently an unknown type of matter that is thought to contain much of the total mass of the universe. Dark matter does not emit or absorb light or electromagnetic or other radiation and therefore cannot be observed directly with telescopes. It is estimated that dark matter makes up $83 \%$ of the matter in the universe and $23 \%$ of its mass-energy. Its existence has not yet been proven experimentally because it does not emit radiation.

For completeness, according to the theories from 2010 , the rest of the matter of the universe consists of:

- Dark energy: About $73 \%$ of the total mass-energy of the universe; it is also a substance, a matter, very little known, only that its name of "energy" is inappropriate

- Baryons: About 5\% - these are the ordinary material world that we perceive directly, including stars, planets, galaxies, etc.

- Neutrinos: About 0.1\%

- Background radiation: Equivalent to about $0.01 \%$ of the matter in the universe

There is theoretical evidence (established by researchers at the University of Pittsburgh, Pennsylvania, USA, based on background radiation - residual radiation from the initial Big Bang explosion) about the existence of dark matter and dark energy, not yet detected. Thus, it was found that the photons in the background radiation are slowed down as they pass through the galaxies more than originally calculated, the delay being due to the 
passage through dark matter. The presence of dark matter is also deduced indirectly from the movement of astronomical objects, especially stars, galaxies and clusters of galaxies ("superclusters").

There are also theories, based on the certainty of the presence of "black holes", which demonstrate the existence of dark matter that is responsible for the accelerated expansion of the universe. For the first time, in our time, a black hole was observed that was caught throwing jets of energy, although it was known that black holes only suck and do not repel matter.

Other theories of the existence of dark matter are based on the gravitational deviations that have been detected regarding the motion of galaxies and clusters of galaxies in the universe, otherwise inexplicable deviations.

The universe is in a permanent expansion that is taking place at a higher speed than space scientists have expected; this speed is imprinted by a curious force called "quintessence" and generated by the cosmic vacuum. The cosmic vacuum, far from being empty, is the seat of unsuspected energies. In the universe, around the black holes are piled up the so-called dark matter, which is so far undetectable, although it is $21 \%$ of cosmic matter.

In early 2007, astronomers drew a three-dimensional map of dark matter that also shows stars and galaxies. The study, published in the journal Nature, provides the most important evidence to date that the spread of galaxies largely corresponds to the distribution of dark matter. The explanation is that dark matter attracts ordinary matter (galaxies, stars, planets, gases, radiation, a total of 5\% of the universe's matter) through the gravitational field.

The constituent particles of dark matter can be neither protons, nor neutrons, nor electrons, nor ordinary neutrinos; cosmologists, who have so far not detected them experimentally, call them, for example, sterile axons and neutrinos. Some brief data on neutrinos can prepare us for what could be dark matter. The neutrino is a stable and very light elementary particle, has no electric charge (so it is electrically neutral) and has a mass at least ten thousand times smaller than that of the electron. The existence of neutrinos was theoretically proved in 1936, they being the explanation for the deviation from the laws of energy conservation; experimentally it was highlighted in 1954 when the first neutrinos were detected.

Day and night we receive from the Sun, every second, almost ten billion neutrinos per square centimeter. It seems that neutrinos do not react with the matter and the interaction with the rest of the universe is weak. The human body is traversed by millions of neutrinos every second. Neutrinos easily cross the volume of our planet, without deviating from the path. Neutrinos change their state frequently. Three types of neutrinos are already known (Hinshaw, 2010; Fritz Zwicky's Biography at the Zwicky Foundation, 2010; The British Universal Encyclopedia, 2010; Trimble, 1987).
Experiments are being carried out with giant accelerators, such as the new LHC accelerator at the CERN European Nuclear Research Center in Geneva, Switzerland and with research, programs involving numerous and particularly powerful scientific forces that are trying to create two bundles of protons that intersect and thus bombard each other. The LHC uses energies of the order of 12 gigajoules and billions of electron-volts. It is expected that in 2009 the conditions existing in the very early period of the Big Bang will be created in the laboratory. During these experiments, it is hoped to discover new elementary particles as well as the mechanisms occurring immediately after the birth of the Universe, in order to create plausible theories of the formation of the universe and the existence and composition of dark matter and dark energy. In addition to the accelerator itself, the experiments use many other special devices, giant computers and special laboratory instruments. They are located underground, on average about $100 \mathrm{~m}$ deep, in a circular tunnel $27 \mathrm{~km}$ long, builtin 2006 near the city of Geneva. In Turin, about $700 \mathrm{~km}$ from Geneva, adequate facilities have been built to receive neutrino beams launched from CERN.

There are already frames recorded on hard disks containing images from particle bombardments performed at enormous energies; printed on paper and stacked on top of each other they would reach the height of the Eiffel Tower. In these frames, phenomena that deviate from the already known physical phenomena also appear extremely rarely. These "anomalies" are intensively studied to uncover the mechanisms that manifested in the early universe, in its early days, immediately after the Big Bang.

Various works aimed to describe the basic structure of moving matter in a dynamic state (Aversa et al, 2017a-b; Duan et al., 2019; Legendi and Bărdescu, 2006; Petrescu and Calautit, 2016a-b; Petrescu et al., 2020; 2019; 2018; 2017a-d; 2016a-b; Petrescu, 2020b, 2019; 2018; 2014; 2012a-b; Petrescu and Petrescu, 2019; 2018; 2014).

Most studies today focus on fusion nuclear energy because it is likely to produce three times more energy than fission, clean, sustainable, safe and without nuclear residues, the reaction being easy to control and on the other hand the fuels needed for fusion are easy to find, water, or heavy water, or the like (Ene, 2010; Kramer, 2011; Moses et al., 2009; Shultis and Faw, 2002; Krane, 1987; Halliday and Robert, 1966).

This paper tries to introduce a new idea and a new theory, according to which opposite particles (of opposite charge) can be obtained other than the antiparticles known so far, with the help of which to produce massive and cheap energy by annihilating them together with normal particles, immediately after the production of the opposite particles (as a load), in order to no longer need to store and keep them for a certain period, difficult operations still with the technologies we have at present. 
The new idea will be presented theoretically, without experiment, because the authors do not yet have the financial possibility to undertake such experiments, even if they do not require large resources or difficult materials and technologies. However, particle accelerations are required at very high energies, which can only be achieved in large linear accelerators.

\section{Attempting to Introduce an Accelerated Electron inside a Neutron}

A proton consists of two quarks up and a quark down, with mass $m_{p}=1,673 \cdot 10^{-27} \mathrm{~kg}$ and positive electric charge $q p=e=1,602 \cdot 10^{-19} \mathrm{C}$, according to Fig. 1 , while a neutron consists of two quarks down and a single quark up, with a mass $m_{n}=1.674927 \cdot 10^{-27} \mathrm{~kg}$ without electric charge as can be seen in Fig. 2.

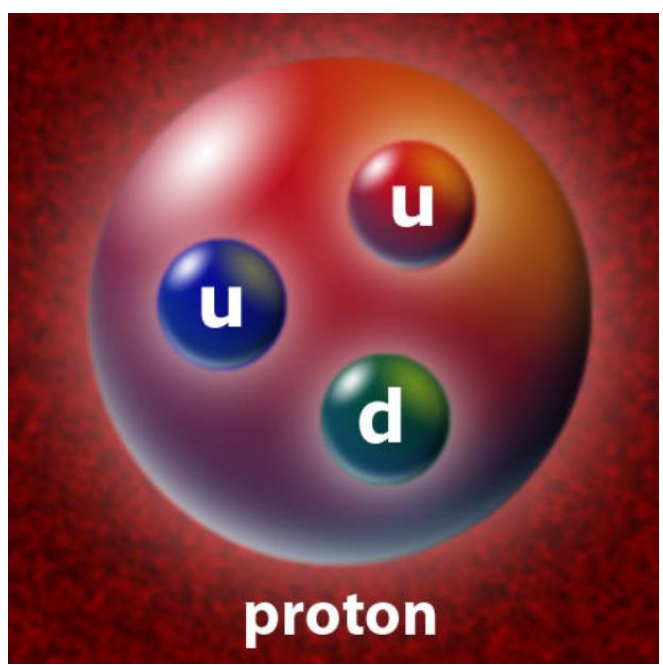

Fig. 1: A proton

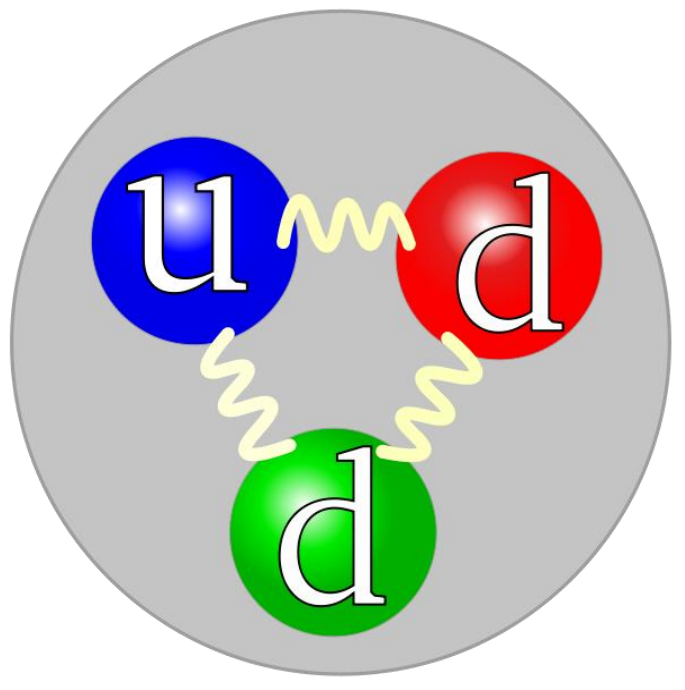

Fig. 2: A neutron
The number of protons is characteristic of all atoms of an element chemical. It represents the number of nuclear charges $\mathrm{Z}$ (the number of positive electrical charges). The number of protons determines the position of the element in Mendeleev's periodic system: The number of protons $=$ the number of nuclear charges $=$ the order number. The proton is symbolized by $\mathrm{p}+$.

Because all the protons of an atom have a positive charge and are all in the nucleus, the question arises why they do not repel, a common physical phenomenon in particles with the same sign of electric charge. The answer is given by the quantum field theory: Protons interact not only by electrostatic force but also by strong nuclear forces. The latter is transmitted by gluons.

Protons were discovered in 1919 by physicist Ernest Rutherford.

The problem of defining the radius of a nucleus is similar to the problem of atomic radius, in the sense that neither atoms nor their nucleus has clear delimitations. However, the nucleus can be represented as a positive charge sphere to analyze the results of electron beam scattering experiments. Because the nucleus has no welldefined limits, electrons "see" a series of effective sections that can be considered an average.

A proton (Fig. 1) is composed of three quarks, two up and one down. Each up quark has a positive charge of $2 / 3 \mathrm{e}+$ and the down quark has a negative charge of $1 / 3 \mathrm{e}-$.

The most logical thing is to position the three quarks of a proton glued together, with no space between them, as we noticed that the whole world of particles is constituted, similar to those in Fig. 3, in which case between the radius of a quark $r$ and that of the proton $R$ appears relation (1):

$$
r=(2 \cdot \sqrt{3}-3) \cdot R_{n}
$$

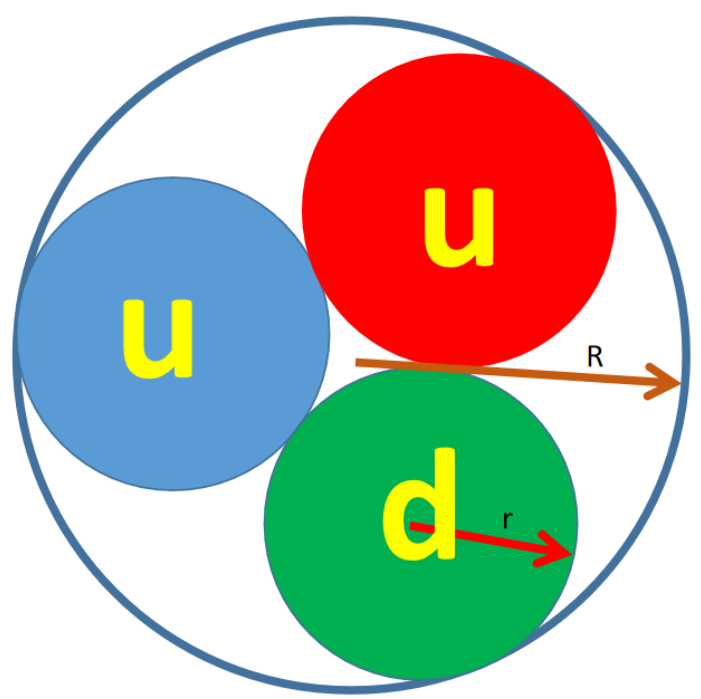

Fig. 3: A proton imagined by the authors 


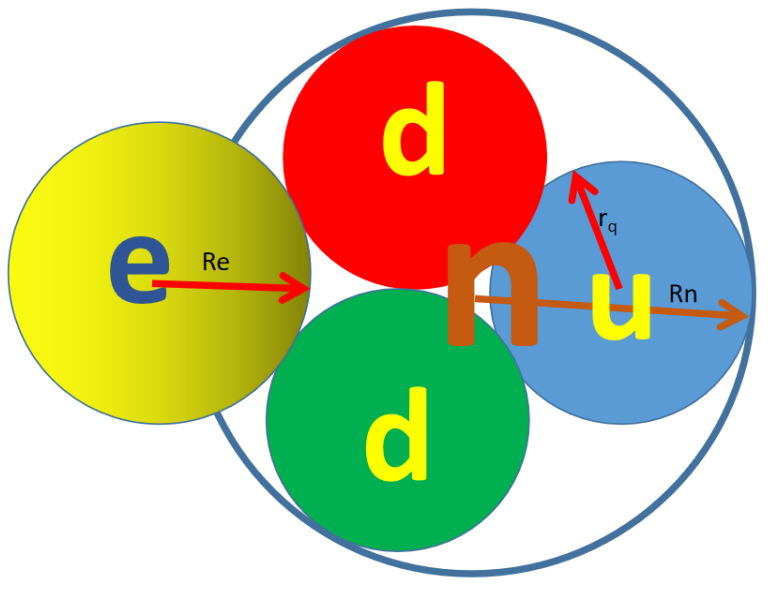

Fig. 4: Electron accelerated to minimum energy that can ensure its adhesion to the two negative quarks of a neutron

In order to try to introduce an electron inside a neutron, in order to obtain in this way a new nuclear particle containing a negative charge, it is necessary to bring the accelerated electron near the two negative quarks (down) of the neutron, without the electron being rejected by the two negative quarks inside the neutron even when they end up glued to each other, ensuring the accelerated electron the minimum energy necessary to penetrate the neutron (Fig. 4).

At this moment the accelerated electron has a minimum acceleration voltage $\mathrm{U}$ according to relation (2). The minimum kinetic energy required for an accelerated electron to penetrate the neutron is calculated by the relation (2), Fig. 4, so that the accelerated electron can stick even to the negative quark, at a distance $d=r_{e}$ considering the radius of the electron $r_{e}\left(R_{e}\right)$ being larger than that of a quark $r$ :

$$
\begin{aligned}
& U_{\min e}[J]=\frac{1}{4 \pi \varepsilon_{0}} \cdot \frac{q_{e} \cdot q_{2 q d}}{r_{e}}= \\
& \frac{1}{4 \pi \cdot 8.8541853 E-12} \cdot \frac{(-) 1.602 E-19 \cdot(-) \frac{2}{3} 1.602 E-19}{R_{e}}
\end{aligned}
$$

The kinetic energy of the accelerated electron is also written in the form (3):

$$
E_{c \min e}[J]=\frac{c^{2} \cdot m_{0 e}}{2} \cdot \frac{\beta^{2}}{\sqrt{1-\beta^{2}}}
$$

The radius of the moving electron can be calculated with the original expression (4) [8, 14-15, 20]:

$$
R_{e}[m]=\sqrt{\frac{10}{8}} \cdot \frac{h \cdot \sqrt{1-\beta^{2}} \cdot \sqrt{1-\frac{1}{2} \beta^{2}-\sqrt{1-\beta^{2}}}}{\pi \cdot m_{0 e} \cdot c \cdot \beta}
$$

Equating the potential energy $U_{\text {mine }}$ with the kinetic energy $E_{c \text { mine }}$ from relations (2) and (3) we obtain the expression of the radius of the accelerated electron (5) depending on the constant $\mathrm{k} 1$ and with relation (6) we express the value of the radius of the accelerated electron taken from the expression (4) at which the constant k3 was also determined:

$$
\begin{aligned}
& R_{e}[m]=k_{1} \cdot \frac{\sqrt{1-\beta^{2}}}{\beta^{2}}=3.7561713 E-15 \cdot \frac{\sqrt{1-\beta^{2}}}{\beta^{2}} \\
& R_{e}[m]=k_{3} \cdot \frac{\sqrt{1-\beta^{2}} \cdot \sqrt{1-\frac{1}{2} \beta^{2}-\sqrt{1-\beta^{2}}}}{\beta}= \\
& 8.6341070 E-13 \cdot \frac{\sqrt{1-\beta^{2}} \cdot \sqrt{1-\frac{1}{2} \beta^{2}-\sqrt{1-\beta^{2}}}}{\beta}
\end{aligned}
$$

Next, the expressions (5) and (6) are equalized and the equation obtained in Beta is processed, in the form (7), where $y=\operatorname{Beta}^{2}$ :

$$
y^{4}+4 \cdot \frac{k_{1}^{2}}{k_{3}^{2}} \cdot y^{2}-8 \cdot \frac{k_{1}^{2}}{k_{3}^{2}} \cdot y+4 \cdot \frac{k_{1}^{4}}{k_{3}^{4}}=0
$$

\section{Results and Discussion}

Equation (7) is solved accordingly, looking for the correct solution that corresponds to both initial relations, (5) and (6) and the following values are obtained:

$\mathrm{Y}=$ beta $^{2}=0.052821885 ;$ beta $=$
$0.229830122 ; \operatorname{Re}[\mathrm{m}]=6.9206569 \mathrm{E}-14 ;$
$\mathrm{ve}[\mathrm{m} / \mathrm{s}]=68901346.86 ;$ Ecmine $[\mathrm{J}]=$
$2.2219177 \mathrm{E}-15 ; \quad$ Ecmine $[\mathrm{eV}] \quad$
$545703617.743 ;$ Ecmine $[\mathrm{GeV}]=0.5457$.

Now the minimum acceleration value of the electron was determined, meant to penetrate a neutron in an attempt to transform it into a particle opposite to the proton, which would annihilate with a proton, thus obtaining a large amount of energy.

The configuration of the new nuclear particle obtained is a strange one, consisting of three down quarks, which together make a whole negative electric charge, equal to that of an electron, although we have a nucleon particle with all the peculiarities of a proton but with the opposite charge (Fig. 5).

If this new theory proposed by this paper comes true, then it opens a new era in the manipulation of nuclear particles and energy.

In order for the penetration reaction to being successful, one can also try to accelerate the neutrons introduced into the enclosure, knowing that neutrons become very active when they are accelerated to a substantial potential. 


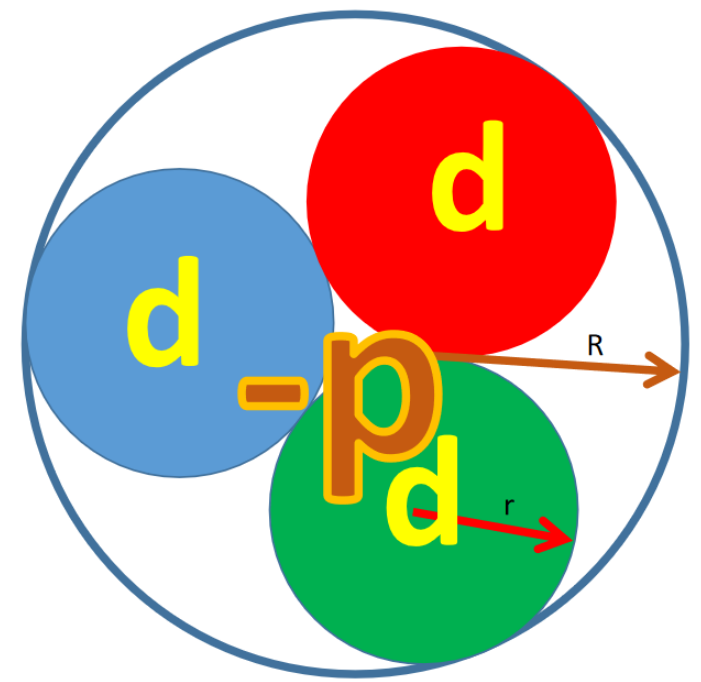

Fig. 5: A negative proton nucleon

It can also be observed that the accelerated electron is still much larger than that of a neutron, which would seem to prevent penetration even if the approach can now occur, but due to the fact that the electron generally has a rarer consistency, it is it can still shrink so much that it can fit inside a neutron. If further acceleration is needed for this or we cannot find out only through physical experiments, so it is not necessary to draw conclusions now about this phenomenon because an electron close to a nuclear particle (a nucleon) has the ability to react with it and even penetrate it due to electromagnetic and nuclear attractions, once the nuclear repulsive forces have been defeated by the energy of the accelerated electron.

The bravest part of the work will be to assume the possibility of obtaining a new nuclear particle in the form of a negative proton.

\section{Conclusion}

The paper aims to present a new theoretical tool capable of helping specialists in the field of nuclear engineering to develop technological systems capable of initiating nuclear reactions and or thermonuclear in good efficiency, on an industrial scale.

The basic ideas of the paper are to obtain nuclear energy by striking accelerated neutrons and electrons at a certain required energy, accelerated electrons so that they can penetrate neutrons and they also transform them into accelerated negative protons so that although the fuel used will be only simple ionized hydrogen (accelerated hydrogen protons) and accelerated neutrons also appear in the system that can improve the efficiency of the nuclear fusion reaction.

The attempt to obtain energy by annihilating opposite elementary particles as a charge is considered.

\section{Acknowledgement}

This text was acknowledged and appreciated by Dr. Veturia CHIROIU Honorific member of Technical Sciences Academy of Romania (ASTR) Ph.D. supervisor in Mechanical Engineering.

\section{Funding Information}

Research contract: Contract number 36-5-4D/1986 from 24IV1985, beneficiary CNST RO (Romanian National Center for Science and Technology) Improving dynamic mechanisms internal combustion engines.

!All these matters are copyrighted!

Copyrights:

- New Aircraft (New Ionic or Beam Engines): no. 548 of 22-04-2010 [cgiywDssin], Aerospace Engineering

- Some Few Specifications About the Doppler Effect to the Electromagnetic Waves: 636 of 28-05-2010 [iEtcaouxxA], physics

- Presenting an Atomic Model and Some Possible Applications in LASER Field: nr. 639 of 29-052010 [yncngrotfo], physics

- Some Applications in LASER Field: no. 718 of 0907-2010 [xeujouincC], physics

- The Energies of Today and Tomorrow: nr. 819 of 30-09-2010 [kbHquxwykr], energy engineering

- Obtaining Energy by the Annihilation of the Matter with Antimatter - The Battle for Energy: nr. 1068 of 13.03.2011 [GfEqpGDzeh], Energy Engineering

\section{Author's Contributions}

All the authors contributed equally to prepare, develop and carry out this manuscript.

\section{Ethics}

This article is original and contains unpublished material. Authors declare that are not ethical issues and no conflict of interest that may arise after the publication of this manuscript.

\section{References}

Aversa, R., Petrescu, R. V., Apicella, A., Kozaitis, S., Abu-Lebdeh, T., Akash, B., \& Petrescu, F. I. (2017a). Triton for nuclear fusion. American Journal of Engineering and Applied Sciences, 10(4).

Aversa, R., Petrescu, R. V., Apicella, A., \& Petrescu, F. I. (2017b). Modern transportation and photovoltaic energy for urban ecotourism. Transylvanian Review of Administrative Sciences, Special, (2017), 5-20. 
Choi, C. Q. (2008). Source of mysterious antimatter found. Space.com. https://www.space.com/4837source-mysterious-antimatter.html

Duan, Y., Zhang, H., Sfarra, S., Avdelidis, N. P., Loutas, T. H., Sotiriadis, G., ... \& Maldague, X. P. (2019). On the Use of Infrared Thermography and AcoustoUltrasonics NDT Techniques for Ceramic-Coated Sandwich Structures. Energies, 12(13), 2537.

Ene, V. M. (2010). Mini Nuclear Power Plant. Fusion reactors. Constructive solutions, impact on the environment. Work gr. Didactic 1, UTCB, 2010.

Fritz Zwicky's Biography at the Zwicky Foundation (2010). http://www.zwicky-stiftung.ch/BioLang.htm

Halliday, D., \& Robert, R. (1966). Physics, Part II. 1st (Edn.)

Hinshaw, G. F. (2010). What is the universe made of? Universe 101 . NASA Website. https://map.gsfc.nasa.gov/universe/uni_matter.html

Kramer, D. (2011). DOE looks again at inertial fusion as a potential clean-energy source. Physics today, 64(3), 26.

Krane, K. S., \& Halliday, D. (1987). Introductory nuclear physics.

Legendi, A., \& Bărdescu, I. (2006). Nanotechnology in support of unconventional energy resources Carbon and porphyrin nanotubes. AROTEM Monitor 3/2006.

Moses, E. I., Boyd, R. N., Remington, B. A., Keane, C. J., \& Al-Ayat, R. (2009). The National Ignition Facility: Ushering in a new age for high energy density science. Physics of Plasmas, 16(4), 041006.

Petrescu, F. I. T. (2012a). Cold nuclear fusion. Plasma Physics and Fusion Technology (S70), INIS 44(16).

Petrescu, F. I. T. (2012b). Cold Nuclear Fusion. Create Space publisher, USA.

Petrescu, F. I. T. (2014). Nuclear Fusion. Infinite Energy. 20(1), 44-47.

Petrescu, F. I. (2018). About the triton structure. American Journal of Engineering and Applied Sciences, 11(4), 1293-1297.

Petrescu, F. I. (2020a). A Hypothesis Which Supports the Possibility of the Existence of Dark Matter with Negative Mass. American Journal of Applied Sciences, 17, 1-5.

Petrescu, F. I. T. (2020b). What is Better for Fusion? American Journal of Applied Sciences. 17(1), 83-87.

Petrescu, F. I. T. (2019). About the nuclear particles' structure and dimensions. Computational Particle Mechanics, 6(2), 191-194.

Petrescu, F. I., \& Calautit, J. K. (2016a). About nano fusion and dynamic fusion. American Journal of Applied Sciences, 13(3).

Petrescu, F. I., \& Calautit, J. K. (2016b). About the light dimensions. American Journal of Applied Sciences, 13(3).
Petrescu, F. I., \& Petrescu, R. V. (2014). Nuclear green energy. Iraqi Journal of Applied Physics, 10(1), 3-14.

Petrescu, N., \& Petrescu, F. I. (2018). Elementary structure of matter can be studied with new quantum computers. American Journal of Engineering and Applied Sciences, 11(2), 1062-1075.

Petrescu, F. I. T., \& Petrescu, R. V. V. (2019). Nuclear hydrogen structure and dimensions. International Journal of Hydrogen Energy, 44(21), 10833-10837.

Petrescu, F. I., Apicella, A., Petrescu, R. V., Kozaitis, S., Bucinell, R., Aversa, R., \& Abu-Lebdeh, T. (2016a). Environmental protection through nuclear energy. American Journal of Applied Sciences, 13(9), 941-946.

Petrescu, R., Aversa, R., Shuhui, L., Chen, G., APICELLA, A., Mirsayar, M., \& Petrescu, T. (2016b). Something about electron dimension.

Petrescu, R. V. V., Machín, A., Fontánez, K., Arango, J. C., Márquez, F. M., \& Petrescu, F. I. T. (2020). Hydrogen for aircraft power and propulsion. International Journal of Hydrogen Energy.

Petrescu, R. V., Aversa, R., Apicella, A., \& Petrescu, F. I. (2018). Modern propulsions for the aerospace industry. American Journal of Engineering and Applied Sciences, 11(2), 715-755.

Petrescu, R. V., Aversa, R., Apicella, A., Abu-Lebdeh, T., \& Petrescu, F. I. (2019). A New Hypothesis About the Nuclear Hydrogen Structure. Available at SSRN 3482167.

Petrescu, R. V., Aversa, R., Li, S., Bucinell, R., Kozaitis, S., Abu-Lebdeh, T., ... \& Petrescu, F. I. (2017a). Electron dimensions. American Journal of Engineering and Applied Sciences, 10(2), 584-602.

Petrescu, R. V., Aversa, R., Kozaitis, S., Apicella, A., \& Petrescu, F. I. (2017b). Deuteron dimensions. American Journal of Engineering and Applied Sciences, 10(3).

Petrescu, R. V., Aversa, R., Kozaitis, S., Apicella, A., \& Petrescu, F. I. (2017c). Some proposed solutions to achieve nuclear fusion. American Journal of Engineering and Applied Sciences, 10(3).

Petrescu, R. V., Aversa, R., Kozaitis, S., Apicella, A., \& Petrescu, F. I. (2017d). Some basic reactions in nuclear fusion. American Journal of Engineering and Applied Sciences, 10(3).

Shultis, J. K., \& Faw, R. E. (2002). Fundamentals of Nuclear Science and Engineering-Kansas State University-Manhattan.

The British Universal Encyclopedia. (2010). Ed. Litera, Bucharest.

Trimble, V. (1987). Existence and nature of dark matter in the universe. Annual review of astronomy and astrophysics, 25(1), 425-472. 
Relly Victoria Virgil Petrescu and Florian Ion Tiberiu Petrescu / American Journal of Engineering and Applied Sciences 2020 , 13 (3): 523.530 DOI: 10.3844/ajeassp.2020.523.530

\section{Nomenclature}

$\mathrm{h}$

$\Rightarrow$ The Planck constant: $h=6.626 \mathrm{E}-34$ [Js]

q

$\Rightarrow$ Electrical elementary load: qe = $1.6021 \mathrm{E}-19[\mathrm{C}] \mathrm{qp}=+1.6021 \mathrm{E}-19[\mathrm{C}]$

c

$=$ The light speed in vacuum: $\mathrm{c}=$ $2.997925 \mathrm{E}+08[\mathrm{~m} / \mathrm{s}]$

The permissive constant (the permittivity): $\varepsilon_{0}=8.85418 \cdot 10^{-12}\left[\frac{C^{2}}{N \cdot m^{2}}\right]$

$\mathrm{n}=$ The principal quantum number (the Bohr quantum number);

$\mathrm{Z}=$ The number of protons from the atomic nucleus (the atomic number);

$\mathrm{m} 0[\mathrm{~kg}] \quad \Rightarrow$ The rest mass of one particle m0electron $=9.11 \mathrm{E}-31[\mathrm{~kg}]$

m0proton $=1.672621898(21) \mathrm{E}-27[\mathrm{~kg}]$

m0neutron $=1.674927471(21) \mathrm{E}-27[\mathrm{~kg}]$

m0deuteron $=\quad 3.34449 \mathrm{E}-27[\mathrm{~kg}]$

m0triton $=5.00827 \mathrm{E}-27[\mathrm{~kg}]$

\section{Source of Figures}

Fig. 1

https://www.universetoday.com/56013/proton-parts/

Fig. 2

https://en.wikipedia.org/wiki/Neutron\#/media/File:Q uark_structure_neutron.svg

Fig. 3-5

Made by authors. 